Artículo de investigación Registro 335762. * Proyecto: "Modelo para la evaluación de la gestión del conocimiento de la universidad (MEGCU): caso de las universidades cofinanciadas de la República del Ecuador".

Consejo Nacional de Ciencia y Tecnología (CONACYT-Méjico) Universidad Nacional Autónoma de México. Recibido: 08.03.2020. Aprobado versión final: 03.06.2020. JEL: I23. Pp. 109-130 * doi: 10.33571/teuken.v11n17a6

\title{
La gestión del conocimiento de las universidades cofinanciadas del Ecuador (UCE)
}

\author{
The knowledge management of the co-financed \\ universities of Ecuador (UCE)
}

\section{Gabriela Carla Cuadrado Barreto ECUADOR}

\begin{abstract}
Resumen: La universidad realiza las funciones de enseñanza, investigación y vinculación con el entorno, por lo que el proceso de evaluación institucional de la gestión del conocimiento y del capital intelectual es indispensable para conocer las actividades que ejecuta. La investigación es de tipo cuantitativa, transeccional, correlacional, no experimental, el contexto de prueba es las Universidades Cofinanciadas del Ecuador (UCE), responde al ¿cómo estudiar los procesos de gestión del conocimiento que realiza la universidad? y propone probar la utilidad del Modelo para la Evaluación de la Gestión del Conocimiento de la Universidad (MEGCU). El alfa de Cronbach alcanza 0.95 y el análisis factorial exploratorio muestra a los procesos particulares de creación, transferencia/almacenamiento y aplicación/uso de conocimiento como variables latentes que explican las actividades que realiza el conjunto de las UCE.
\end{abstract}

Palabras clave: evaluación universitaria; gestión del conocimiento; capital intelectual; educación superior.

\begin{abstract}
The university realizes the functions of teaching, research and linkage with the environment, so the process of institutional assessment of knowledge management and intellectual capital is essential to understand the activities it implements. The research is quantitative, transectional, correlational, and nonexperimental. The testing context is the Co-Funded Universities of Ecuador (UCE), which responds to the question of how to study the knowledge management processes carried out by the university and proposes to demonstrate the usefulness of the Model for the Assessment of University Knowledge Management (MEGCU). Cronbach's alpha reaches 0.95 and the exploratory factorial analysis shows the particular processes of creation, transfer/storage and application/ use of knowledge as latent variables that explain the activities carried out by the group of UCE.
\end{abstract}

Keywords: assess of university, knowledge management, intellectual capital, model

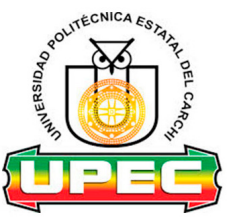

Gabriela Cuadrado-Barreto es Doctora en Ciencias de la Administración por la Universidad Nacional Autónoma de México, Docente Investigador Titular de Tiempo Completo en la Universidad Politécnica Estatal del Carchi (Tulcán-Ecuador), miembro del Grupo de Investigación Frontera Norte, en las líneas de investigación en Gestión del conocimiento Capital intelectual, enseñanza superior, universidad.

Contacto: gabrielacuadradobarreto@gmail.com https://orcid.org/0000-0002-8562-7843. 


\title{
Gestão do conhecimento na universidade co-financiada do Equador (UCE) \\ uma análise exploratória.
}

\begin{abstract}
Resumo: A universidade realiza as funções de ensino, pesquisa e ligação com o meio ambiente, por isso o processo de avaliação institucional da gestão do conhecimento e do capital intelectual é essencial para entender as atividades que ela implementa. A pesquisa é quantitativa, transeccional, correlacional e não-experimental. O contexto de teste é a Universidade Co-Fundada do Equador (UCE), que responde à questão de como estudar os processos de gestão do conhecimento realizados pela universidade e se propõe a demonstrar a utilidade do Modelo de Avaliação da Gestão do Conhecimento Universitário (MEGCU). O alfa de Cronbach atinge 0,95 e a análise factorial exploratória mostra os processos particulares de criação, transferência/armazenamento e aplicação/utilização do conhecimento como variáveis latentes que explicam as atividades realizadas pelo grupo da UCE.
\end{abstract}

Palavras-chave: avaliação universitária; gestão do conhecimento; capital intelectual; ensino superior.

\section{Introducción}

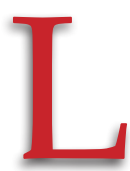

a universidad identificada como el lugar de posibilidades para hacerfrente a los desafíos de temáticas emergentes de la sociedad del conocimiento requiere evaluar su accionar alrededor de la función social que ejecuta (Streck, 2018). Al considerar que un modelo representa fenómenos y situaciones reales para la comprensión del entorno (Ramírez, Manzanaque \& Priego, 2017), la evaluación de la universidad, a través del Modelo para la Evaluación de la Gestión del Conocimiento de la Universidad (MEGCU), tiene el propósito de validar el MEGCU en contextos reales mediante la recolección de las opiniones de los expertos organizacionales y la representación de la causalidad de los procesos de gestión con los resultados de conocimiento.

La universidad aporta el conocimiento, con características científicotecnológicas, en la sociedad al capacitar a los integrantes de las empresas, instituciones sin fines de lucro y entidades de gobierno; transferir la tecnología y preservar la cultura de un país (Gupta, Mehrotra, \&Sharma, 2015; Rinesi, 2018). En concordancia, la evaluación de la institución universitaria permite conocer el estado actual de la organización al determinar que el objeto evaluado (organización universitaria) necesita cumplir con la característica de susceptibilidad a la clasificación, con el propósito de lograr comparaciones entre entidades (García-Berro, Roca, Navallas, Soriano, y Ras, 2016; De Sousa, 2015; Medland, 2016; Ramírez, 2016). 
En este sentido, para la presente investigación, la universidad se evalúa para medir los procesos de gestión del conocimiento, los resultados de conocimiento científico tecnológico y la relevancia de las actividades que desempeña para explicar los resultados organizacionales obtenidos por la institución en un tiempo determinado, los propósitos institucionales y planear acciones para la mejora organizacional.

De esta forma, en principio, a través de la metodología de sistemas complejos organizacionales se precisa al Modelo para la Evaluación de la Gestión del Conocimiento de la Universidad (MEGCU). También, se da a conocer la prueba del MEGCU en el contexto de la Universidad Cofinanciada del Ecuador (UCE). Las UCE se identifican como un conjunto de siete universidades con características similares de tamaño, propósitos y tipo de financiamiento. Por último, tras el análisis de los factores principales se dan a conocer las conclusiones alrededor de la gestión del conocimiento y del capital intelectual, como dimensiones del MEGCU, se puntualiza al modelo propuesto como útil al medir de forma adecuada y precisa tanto al conjunto de universidades, como de forma individual a la institución universitaria.

\section{Perspectiva metodológica y método de investigación}

La investigación se aborda desde la perspectiva de la complejidad organizacional (Lara, 2017; Ostrom, 2015) con el propósito de probar la utilidad del objeto complejo denominado Modelo para la Evaluación de la Gestión del Conocimiento de la Universidad (MEGCU). El método es cuantitativo, corresponde a una investigación exploratoria, de tipo no experimental, con un diseño transeccional y correlacional (Creswell, 2014). La indagación se realiza en el contexto de la Universidad Cofinanciada del Ecuador (UCE), integrada por siete instituciones universitarias y en el estudio participan 100 directores de carrera identificados como directivos de nivel medio de la organización. Además, los resultados son analizados mediante técnicas de análisis factorial, método de extracción de componentes principales, coeficiente Tucker, método de Torgerson y análisis multivariante $\mathrm{Hj}$-Biplot con las herramientas estadísticas SPSS V.22 y MultiBiplot para explicar la realidad organizacional de las instituciones de educación superior. Así, se detalla los resultados de conocimiento generados, los procesos de gestión del conocimiento específicos desarrollados, la función perseguida por las UCE y se ultima al MEGCU como un aporte teórico útil para indagar el sistema complejo de las actividades de la universidad. 


\section{Modelo para la Evaluación de la Gestión del Conocimiento de la Universidad (MEGCU)}

Al considerar que las funciones que realiza la organización universitaria se encuentran soportadas por procesos organizacionales, el MEGCU integra la Gestión del conocimiento (GC) y el Capital intelectual (Cl) como variables de estudio. A continuación, se detallan los elementos del modelo propuesto.

La GC desde la Teoría de Creación del Conocimiento Organizacional (TCO) se identifica como un proceso social, continuo y dinámico que permite la coherencia entre la estructura organizacional con la ejecución de las actividades que realizan los miembros de la organización (Barley, Treem \& Kuhn, 2018; Cuadrado y Carrillo, 2016; Henríquez, 2018; Nonaka \& Takeuchi, 1995; Sadeghi, Hasani \& Delshab, 2019). A su vez, la GC se compone de los procesos particulares de creación (CC), transferencia/almacenamiento (TA) y aplicación/uso (AU) del conocimiento, establecidos como dimensiones del MEGCU. Así, la ejecución de estos procesos particulares aporta a la generación de resultados en la universidad y el proceso de evaluación de la institución universitaria se convierte en imperante por precisarse a esta organización, la universidad, como eje fundamental de la sociedad del conocimiento.

La creación de conocimiento (CC) se define como un proceso particular de tipo social en el que interactúan los individuos en el contexto universitario, promueve el desarrollo de diferentes formas de conocimiento e integra la adquisición, interpretación y diseminación del conocimiento (Serenko, 2019; Abbas, Avdic, Xiaobao, Hasan \& Ming, 2019; De Silva \& Rossi, 2018; Serenko \& Bontis, 2016). La transferencia/ almacenamiento del conocimiento (TA) sostiene el desarrollo de las funciones de enseñanza, investigación, vinculación con la sociedad y difusión de la cultura que ejecutan los integrantes de la universidad mediante procedimientos establecidos y el uso de tecnologías de la información para la administración del conocimiento humano codificado como activo intangible (Ferreira, Mueller \& Papa, 2018; Marchiori \& Franco, 2019; Azagra-Caro, BarberáTomás, Edwards-Schachter \&Tur, 2016). La aplicación/ uso de conocimiento (AU) contribuye a la resolución de problemas de la sociedad al integrar el compromiso con el conocimiento, trabajo en equipo y la apropiación del conocimiento; lo que permite a los integrantes de la organización establecer soluciones innovadoras a los problemas (Baglieri, Baldi \& Tucci, 2018; Elezi \& Bamber, 2018; Bratianu, 2018a).

De esta forma, la ejecución del proceso particular TA permite que el conocimiento de la universidad se encuentre a disposición de sus integrantes para el desarrollo de las actividades; la CC permite la 
combinación de los conocimientos ya existentes en la organización; y el AU favorece el desarrollo de la función social de la institución.

La universidad, al reconocerse como fuente generadora de conocimiento en la sociedad encuentra la misión de mostrar el resultado organizacional de activos de conocimiento, provenientes de los procesos de gestión que realiza, denominados como capital intelectual (CI) (Etzkowitz, 2017; Bejinaru, 2017a; Miake, Carvalho, Pinto \& Graeml, 2018). El Cl de la universidad desde la Teoría de Recursos y Capacidades de la Administración (TRC) se define como el resultado del proceso de producción del conocimiento desarrollado durante un período de tiempo determinado (Frondizi, Fantauzzi, Colasanti \& Fiorani, 2019; Bejinaru, 2018; Askary, Qayyum \& Van Sant, 2015), se compone de capital humano $(\mathrm{CH})$, capital estructural (CE) y capital relacional (CR), y se identifican como dimensiones del modelo MEGCU.

El capital humano $(\mathrm{CH})$ muestra el conocimiento, las destrezas y habilidades, la experiencia, la innovación y creatividad del personal universitario, tales como profesores, investigadores, directivos universitarios, personal de administración y servicios (Zharova, 2018; Bratianu, 2018b; Nava, Cernas y Jiménez, 2016). El capital estructural (CE) se identifica como exclusivo de la universidad por lo que incorpora la infraestructura física, los sistemas informáticos, los procesos, la estructura y cultura de la organización y la propiedad intelectual (Blind, Pohlisch, \& Zi, 2018; Demuner, Nava \& Ibarra, 2016). El capital relacional (CR) recoge un amplio conjunto de relaciones económicas, institucionales y políticas, desplegadas y mantenidas con socios tanto académicos como no académicos que integra proveedores, vinculación con la sociedad, reputación, redes de trabajo (Ramírez \& Manzaneque, 2015; Mercado, García y Cernas, 2016a; Crescenzi, Nathan \& Rodríguez-Pose, 2016).

Así, entre los elementos de la institución universitaria el capital humano da a conocer el conocimiento formal e informal que poseen los integrantes de la universidad para la ejecución de sus actividades, el capital estructural proporciona acceso a los activos de conocimiento explícito y el capital relacional promueve la difusión de los resultados de investigación, la difusión de la cultura y la movilidad de los universitarios.

A su vez, el MEGCU, como objeto de estudio complejo, a través de los principios de diversidad e interdependencia integra a los procesos particulares de GC - CC, TA, AU-y a los elementos del capital intelectual - $\mathrm{CH}, \mathrm{CE}, \mathrm{CR}$ - como dimensiones que permiten la evaluación de las acciones de la universidad con características de sistema abierto complejo y que integra elementos en constante interacción. A su vez, el MEGCU se articula con el Cuestionario para la evaluación de la gestión 
del conocimiento (CEGCU) que presenta trece escalas, 80 ítems y como encuesta de tipo autoadministrada permite la recolección de datos alrededor de la gestión del conocimiento y el capital intelectual de la universidad (véase tabla 1).

De esta forma, los resultados del proceso de GC se establecen a través de escalas de tipo diferencial semántica que involucra los niveles: a) Inicial, con procesos desorganizados; b) Regular, con procesos que siguen un patrón de ejecución; c) Definido, con procesos estandarizados en la organización; d) Administrado, con procesos integrados y monitoreados por la institución universitaria; e) Optimizado, con procesos depurados de tipo mejores prácticas (Cuadrado, 2017).

Tabla 1 Elementos del Modelo para la Evaluación de la Gestión del Conocimiento de la Universidad (MEGCU)

\begin{tabular}{|c|c|c|}
\hline Variables & Dimensiones & Escalas \\
\hline \multirow{8}{*}{ 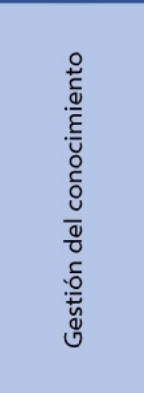 } & \multirow{3}{*}{$\begin{array}{l}\text { Creación de conocimiento } \\
\text { (CC) }\end{array}$} & Interpretación \\
\hline & & Diseminación de la información \\
\hline & & Adquisición de información \\
\hline & \multirow{2}{*}{$\begin{array}{l}\text { Transferencia/ } \\
\text { almacenamiento } \\
\text { de conocimiento (TA) }\end{array}$} & Transferencia \\
\hline & & Almacenamiento \\
\hline & \multirow{3}{*}{$\begin{array}{l}\text { Aplicación/ uso (AU) } \\
\text { del conocimiento }\end{array}$} & Trabajo en equipo \\
\hline & & Apropiación del conocimiento \\
\hline & & Compromiso con el conocimiento \\
\hline \multirow{12}{*}{ 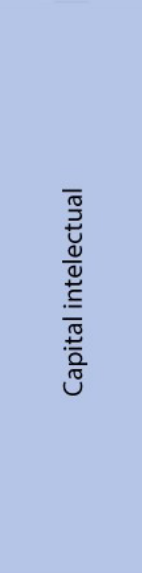 } & \multirow{3}{*}{ Capital humano } & Conocimiento, destrezas y habilidades \\
\hline & & Experiencia \\
\hline & & Innovación y creatividad \\
\hline & \multirow{5}{*}{ Capital estructural } & Infraestructura física \\
\hline & & Sistemas informáticos \\
\hline & & Cultura organizacional \\
\hline & & Estructura organizacional \\
\hline & & Propiedad intelectual \\
\hline & \multirow{4}{*}{ Capital relacional } & Proveedores \\
\hline & & Vinculación con la sociedad \\
\hline & & Reputación \\
\hline & & Redes de trabajo \\
\hline
\end{tabular}

Fuente: Elaboración propia.

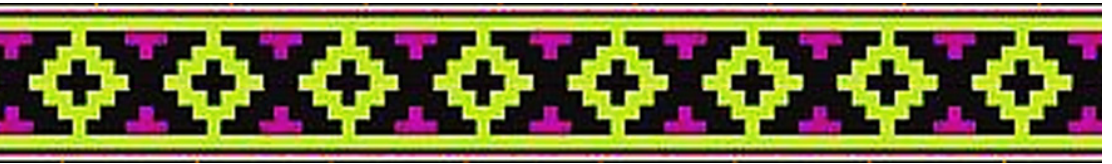




\section{Resultados y Discusión}

La investigación encuentra el propósito de probar la utilidad del objeto complejo denominado Modelo para la Evaluación de la Gestión del Conocimiento de la Universidad (MEGCU). El MEGCU se valida en el contexto de la Universidad Cofinanciada del Ecuador (UCE) , integrado por siete instituciones que, por primera vez, evalúan su gestión del conocimiento y capital intelectual de la organización. Estas instituciones se encuentran las tres principales ciudades del país - Quito, Guayaquil, Cuenca-, especifican objetivos comunes de enseñanza, investigación, vinculación y el principio católico, gozan de autonomía institucional, libertad tanto académica, como de pensamiento, con estudiantes de clase media en su mayoría (Cordero, 2016).

En pro de garantizar la factibilidad y viabilidad de acceso a los datos, se solicitó a las autoridades institucionales el permiso para encuestar a los informantes universitarios. La estructura organizacional de las UCE muestra a las facultades y las carreras por disciplinas científicas humanidades, ciencias sociales, ciencias, ingeniería y salud-, por lo que se considera como informantes de calidad a los directores de carrera por disciplina científica como miembros de nivel táctico de la organización universitaria. Al meditar que el tamaño de la muestra -100 directores de carrera-cumple con el propósito de poseer un número potencial de candidatos que proporcionen información de calidad para la investigación (Mochales, 2014). Así, se realiza un muestreo no probabilístico que combina muestreo intencional, muestreo tipo bola de nieve y por conveniencia (Creswell, 2014) para comprobar la hipótesis: El Modelo para la Evaluación de la Gestión del Conocimiento de la Universidad (MEGCU) evalúa y mide los procesos de gestión del conocimiento (GC) y los elementos del capital intelectual que posee la universidad.

\section{La gestión del conocimiento en el conjunto de UCE}

El análisis exploratorio de los datos del conjunto de UCE se realiza a través del Coeficiente de Variación de Pearson (CVP), de la media, la desviación típica y la varianza, para el análisis de las dimensiones correspondientes a la GC y al Cl. Así, el capital humano, el capital estructural y el capital relacional presentan varianzas cero o muy cercanas a cero, los porcentajes del CVP son también cero o extremadamente bajos, y no suponen ninguna contribución significativa en la formación de grupos diferenciados.

El análisis factorial exploratorio se analiza con el coeficiente KMO que indica 0.892, la Prueba de esfericidad de Bartlett resulta en un Chicuadrado aproximado de 3454,086 , así las dimensiones correspondientes 
a la variable GC presentan un alto nivel de relación. El método de extracción de componentes principales muestra, a través del gráfico de codo, a los 41 ítems que conforman las dimensiones de creación de conocimiento (CC), transferencia/ almacenamiento de conocimiento (TA) y aplicación/ uso de conocimiento (AU) de la GC (véase gráfica 1). Además, el método de rotación Varimax da a conocer que las dimensiones CC, TA, AU explican el $57.70 \%$ de la variabilidad de los datos. Los métodos Torgerson, Proxscal, la medida de Minkowski y el coeficiente de Tucker permiten calcular las distancias entre las escalas en un espacio bidimensional (véase tabla 2) y se marca con diferentes colores a los componentes en función de las escalas a las que pertenecen (véase gráfica 2). De esta forma, es posible concluir que, en general, los componentes ocupan zonas bien definidas del mapa, presentan un elevado nivel de cercanía, lo que se traduce en una definición de las tres dimensiones - CC, TA, AU- como variables latentes claramente definidas.

Gráfica 1. Sedimentación del proceso de GC de las UCE

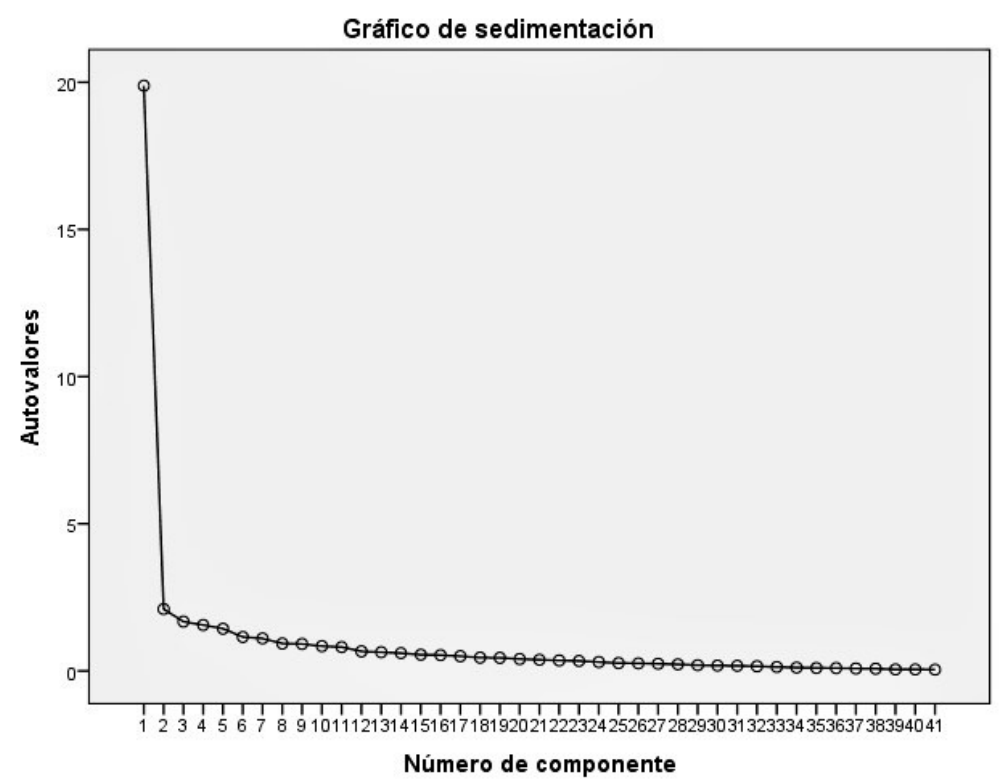

Fuente: Elaboración propia - SPSS v22

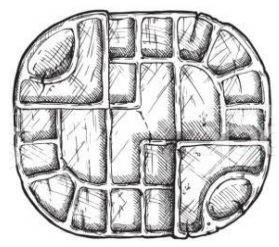


Gráfica 2 Análisis Proxcal del proceso de GC de las UCE

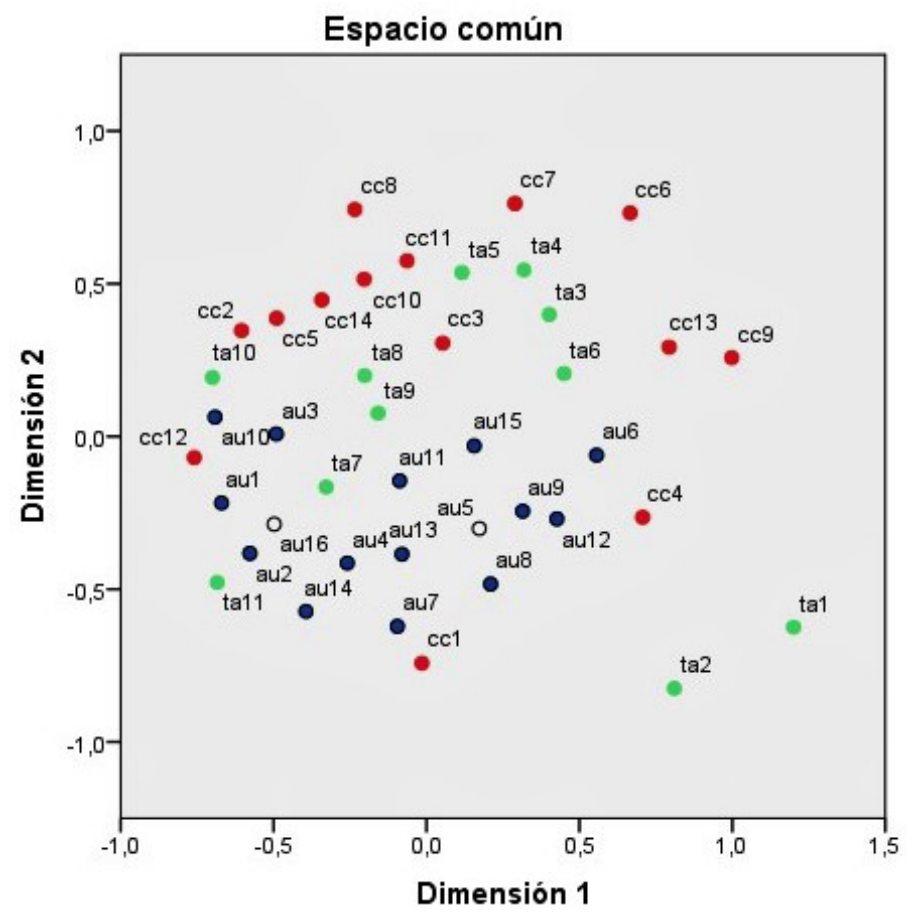

Fuente: Elaboración propia - SPSS v.22.

El análisis multivariante $\mathrm{Hj}$-Biplot se ejecuta mediante el software MultiBiplot (Nieto et al., 2014), las coordenadas muestran tres variables latentes de CC, TA, AU, pertenecientes al proceso de GC, diferenciadas mediante colores se observa una distribución definida de los grupos (véase gráfica 3).

Tabla 2 Medidas de ajuste y stress

\begin{tabular}{|l|r|}
\hline Stress bruto normalizado &, 09754 \\
\hline Stress-I &, $31231^{\mathrm{a}}$ \\
\hline Stress-II &, $72427^{\mathrm{a}}$ \\
\hline S-Stress &, $19033^{\mathrm{b}}$ \\
\hline Dispersión explicada (D.A.F.) &, 90246 \\
\hline Coeficiente de congruencia de Tucker &, 94998 \\
\hline & \\
\hline PROXSCAL minimiza el stress bruto normalizado. \\
\hline a. Factor para escalamiento óptimo $=1,108$. \\
\hline b. Factor para escalamiento óptimo $=, 902$. \\
\hline
\end{tabular}

Fuente: Elaboración propia mediante el uso de SPSS V.22 
Gráfica 3. HJ BIPLOT del Proceso de GC.

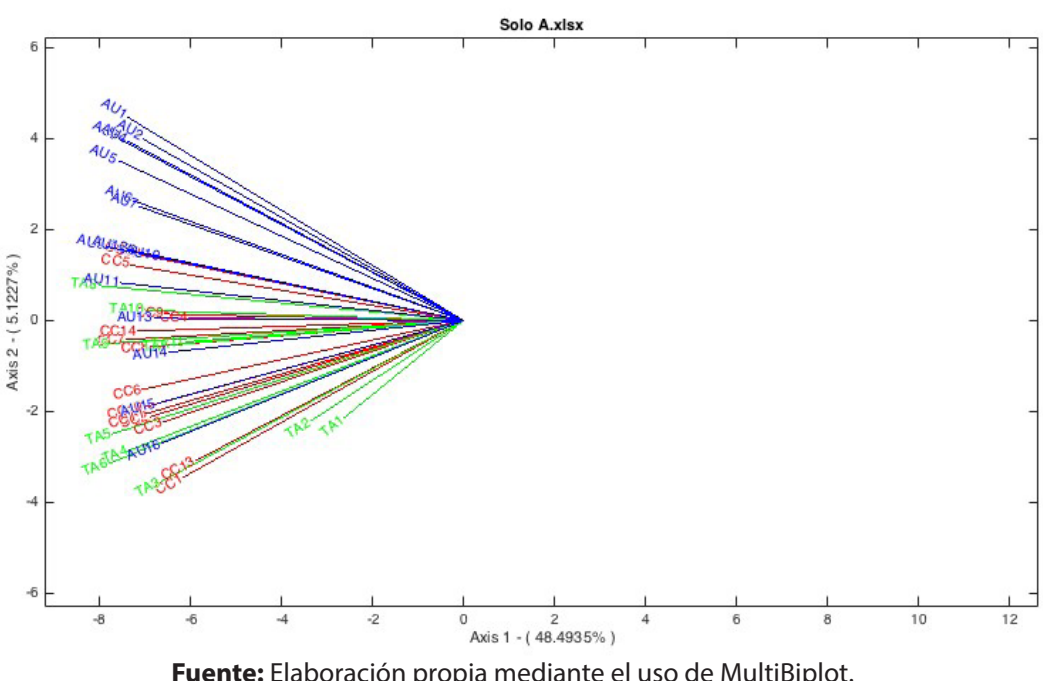

En concordancia con lo anterior, en primera instancia, el proceso particular Aplicación y Uso de conocimiento (AU) se ejecuta en mayor medida, por lo que se encuentra en un nivel de Monitoreado al alcanzar procesos depurados y de mejor práctica en el conjunto de las UCE. A su vez, los componentes que refieren a la actualización continua de las bases de datos con información de los organismos relacionados y la documentación de los procesos organizacionales se encuentran un tanto distantes del conjunto se determina que el proceso se encuentra administrativamente medido y monitoreado.

También, el proceso particular CC explica el $48.49 \%$ de los datos, se ubica en el segundo eje latente, en un nivel estandarizado de gestión, a excepción de los componentes relacionados con el descarte de información obsoleta y el beneficio de la información para la universidad y se determinan como medidos, monitoreados y pertenecen a un nivel integrado del proceso. $\mathrm{Al}$ mismo tiempo, los componentes de recolección de información y ruptura de concepciones tradicionales desde los docentes aparecen alejados del agrupamiento, por motivo de la estandarización, la escasa difusión en la universidad, por hallarse implementados y documentados.

Por último, el proceso particular de transferencia y almacenamiento de conocimiento (TA) expone proximidad entre los componentes de transferencia de conocimiento que involucra el aprendizaje de conocimiento organizacional y de habilidades críticas, de mejora institucional a partir de los nuevos conocimientos adquiridos y el 
considerar a la universidad como una organización de aprendizaje, exhibe la existencia de un nivel estandarizado del proceso al encontrarse implementado y documentado. Los componentes del almacenamiento de conocimiento, aunque alejados del grupo anterior, mantienen un alto nivel de cohesión entre sí, lo que demuestra la integración del proceso en la universidad, a su vez se encuentra administrativamente medido y monitoreado. Además, los componentes de conocimiento como fuente de poder de los docentes y la pérdida de conocimiento por la rotación de docentes se alejan del conjunto de preguntas se encuentran en un nivel inicial (véase tabla 3).

Tabla 3 Niveles de ejecución de los procesos de GC en el conjunto de UCE

\begin{tabular}{|l|c|c|c|c|}
\hline \multicolumn{1}{|c|}{ Escala } & $\begin{array}{c}\text { Alfa de } \\
\text { Cronbach }\end{array}$ & Eje latente & Componentes esenciales & Nivel \\
\hline $\begin{array}{l}\text { Aplicación/ uso (AU) } \\
\text { del conocimiento }\end{array}$ & 0,951 & Primero & $\begin{array}{l}\text { Trabajo en equipo, apropiación } \\
\text { del conocimiento y compromiso } \\
\text { con el conocimiento }\end{array}$ & Monitoreado \\
\hline $\begin{array}{l}\text { Creación } \\
\text { de conocimiento (CC) }\end{array}$ & 0,927 & Segundo & $\begin{array}{l}\text { adquisición, diseminación e } \\
\text { interpretación de la información }\end{array}$ & Estandarizado \\
\hline $\begin{array}{l}\text { Transferencia/ } \\
\text { almacenamiento de } \\
\text { conocimiento (TA) }\end{array}$ & 0,891 & Tercero & Transferencia de conocimiento & Estandarizado \\
\hline
\end{tabular}

Fuente: Elaboración propia.

El análisis anterior corrobora la obtención de hallazgos muy similares con el método de análisis factorial, PROXCAL y HJ-BIPLOT (Galego, Formigoni y Antunes, 2014), la variabilidad medida como la sumatoria de sus valores en los dos ejes explica el 53,61 por ciento de la variabilidad de los datos, así, los ítems de cada escala presentan altos niveles de definición. Por lo que, se demuestra la aplicabilidad del MEGCU a la hora de evaluar las dimensiones, los criterios, las escalas y los componentes de la universidad como un sistema complejo.

\section{El capital intelectual en el conjunto de UCE}

El estudio del Cl se efectúa, en un principio, a través del análisis PROXCAL para identificar la distribución de los elementos de capital humano $(\mathrm{CH})$, capital estructural (CE) y capital relacional (CR) (véase gráfica 4). El capital humano presenta ciertas proximidades, exceptuando los temas relacionados a las publicaciones científicas individuales y a las memorias de participaciones en conferencias y congresos, que por tratarse de responsabilidades individuales de los docentes se ubican en el nivel de gestión de patrón regular. El CE muestra la representatividad de los manuales de procedimientos, lo que ubica a la universidad en el nivel 
inicial de gestión ya que requiere direccionar sus procesos. El valor del estrés bruto normalizado del $\mathrm{Cl}$ es cercano a cero y el valor del coeficiente de congruencia de Tucker se aproxima a 1, lo que indica una buena representación del modelo (véase tabla 4).

Tabla 4 Medidas de ajuste y stress de $\mathrm{Cl}$

\begin{tabular}{|l|r|}
\hline Stress bruto normalizado &, 01277 \\
\hline Stress-I &, $11302^{\mathrm{a}}$ \\
\hline Stress-II &, $14369^{\mathrm{a}}$ \\
\hline S-Stress &, $02806^{\mathrm{b}}$ \\
\hline Dispersión explicada (D.A.F.) &, 98723 \\
\hline $\begin{array}{l}\text { Coeficiente de congruencia } \\
\text { de Tucker }\end{array}$ &, 99359 \\
\hline
\end{tabular}

Fuente: Elaboración propia. SPSS v.22.

Gráfica 4. Análisis PROXCAL del Cl de las UCE

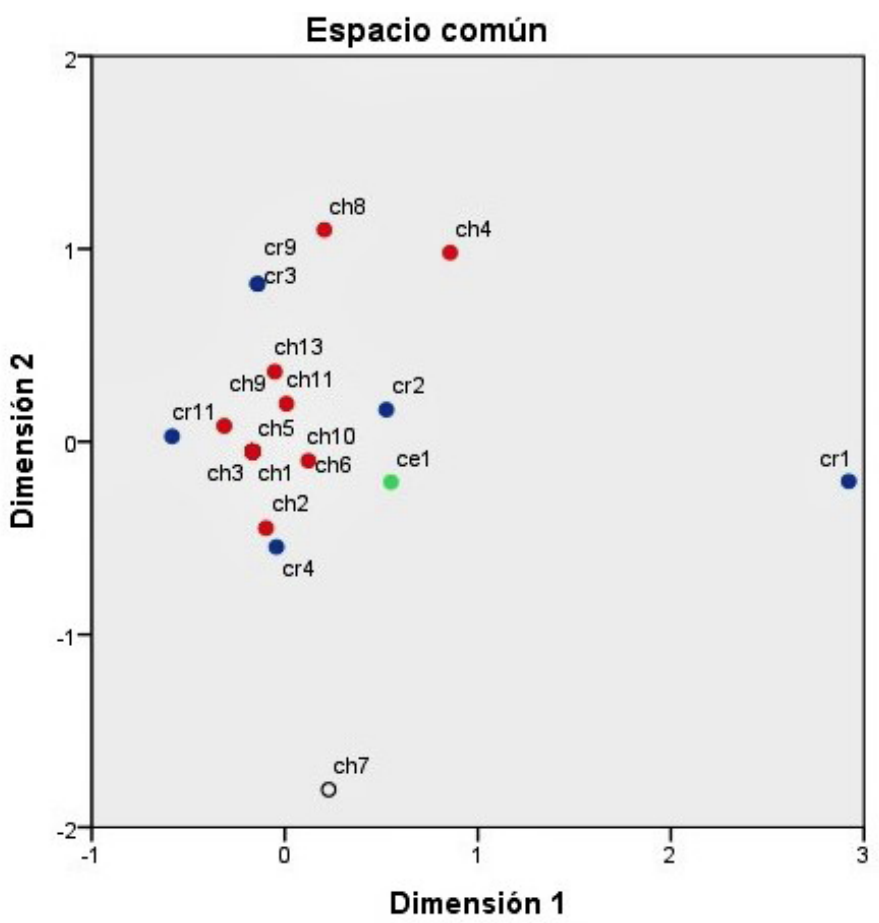

Fuente: Elaboración propia mediante el uso de SPSS V.22. 
Tabla 5 La GC y el Cl en las UCE

\begin{tabular}{|l|c|c|c|}
\hline \multicolumn{2}{|c|}{ Proceso de GC } & \multicolumn{2}{|c|}{ Capital intelectual } \\
\hline item & \multicolumn{1}{|c|}{$\boldsymbol{p}$} & item & \multicolumn{1}{c|}{$\boldsymbol{p}$} \\
\hline CC6 & $0,002^{* *}$ & CH10 & $0,005^{* *}$ \\
\hline CC10 & $0,027^{*}$ & CE1 & $0,023^{*}$ \\
\hline CC13 & $0,037^{*}$ & & \\
\hline TA5 & $0,034^{*}$ & \\
\hline TA6 & $0,004^{* *}$ & \\
\hline TA8 & $0,042^{*}$ & \\
\hline AU6 & $0,025^{*}$ & \\
\hline AU7 & $0,006^{* *}$ & \\
\hline AU8 & $0,005^{* *}$ & \\
\hline AU11 & $0,042^{*}$ & \\
\hline AU12 & $0,002^{* *}$ & \\
\hline AU13 & $0,009^{* *}$ & \\
\hline AU14 & $0,028^{*}$ & \\
\hline AU15 & $0,008^{* *}$ & \\
\hline AU16 & $0,013^{*}$ & \\
\hline & & \\
\end{tabular}

La distribución de los elementos del $\mathrm{CH}, \mathrm{CE}, \mathrm{CR}$, por medio del método de HJ-BIPLOT, ratifica las distribuciones no tan definidas debido a su reducida variabilidad y su baja fiabilidad (véase gráfica 5), así se determina que las UCE se encuentran en un nivel inicial de capital intelectual $(\mathrm{Cl})$ y que requieren el direccionamiento de sus procesos de gestión.

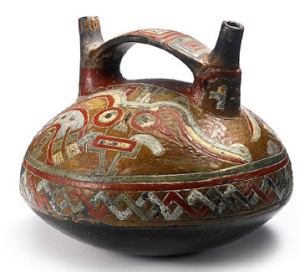

Fuente: Elaboración propia. - MultBiplot.

Gráfica 5. HJ BIPLOT Cl de las UCE

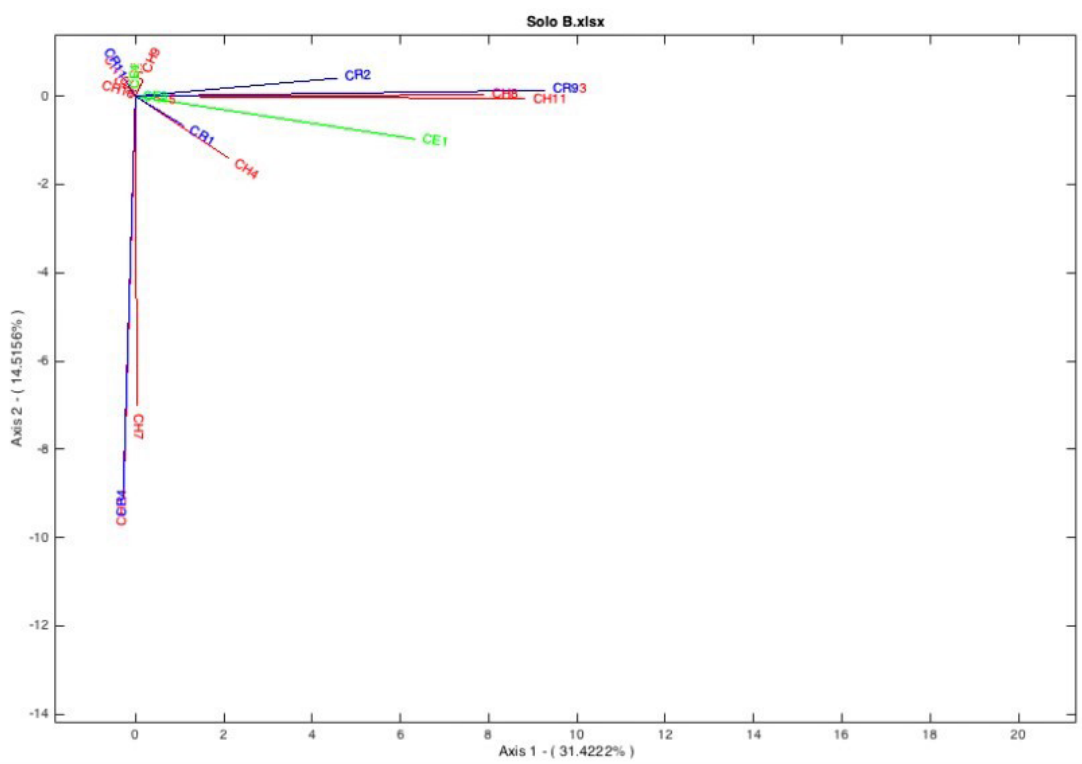

Fuente: Elaboración propia. SPSS v.22 


\section{Nivel de gestión del conjunto de Universidades Cofinanciadas del Ecuador (UCE)}

Al considerar al contexto de prueba del MEGCU como el conjunto de las universidades cofinanciadas del Ecuador (UCE) también se especifican los niveles de gestión que ejecutan las UCE a partir de los elementos altamente correlacionados (véase tabla 5). La GC expone una alta correlación en la creación de conocimiento (CC) ya que la diseminación de la información se encuentra promovida a través de políticas universitarias de compensación para el intercambio de conocimientos. Ello corrobora la relevancia del intercambio de información entre los miembros de la universidad en relación con el cumplimiento de los fines organizacionales (Leih \& Teece, 2016; Alimohammadlou \& Eslamloo, 2016).

La transferencia y almacenamiento de conocimiento (TA) en el conjunto de UCE exhibe que las instituciones conservan procedimientos para la distribución de las propuestas desde la comunidad universitaria para el desarrollo del conocimiento, lo que coincide con Mahdi, Nassar \& Almsafir (2019); Mansor, Mustaffa \& Salleh (2015).

Por último, la aplicación y uso de conocimiento (AU) y su alta correlación con el $\mathrm{Cl}$ permite conocer la importancia que tiene el autocontrol y la autonomía en la toma de decisiones de los docentes, la incorporación de sugerencias de estudiantes, los mecanismos para promover la creación de conocimiento y la actualización de bases de datos como directriz a seguir en las UCE. Este hallazgo refuerza el supuesto de que la apropiación y fomento del compromiso con el conocimiento de los integrantes de la universidad producen resultados de conocimiento organizacional (Muscio \& Ramaciotti, 2019; Olcay \& Bulu, 2017). Además, la diseminación y la interpretación de la información y el compromiso con el conocimiento se consideran promotores, en menor medida, de los resultados organizacionales del conocimiento en la organización universitaria, que coincide con Petrova, Smokotin, Kornienko, Ershova \& Kachalov (2015); Schaik, Volman, Admiraal \& Schenke (2018); Sengupta \& Ray (2017).

Como parte del $\mathrm{Cl}$, el capital humano $(\mathrm{CH})$ refiere a las estancias de investigación en el extranjero, con una alta correlación se reconoce que la universidad el dar a conocer la ejecución de procesos de GC genera experiencia para el desarrollo de la investigación (Bejinaru, 2017b; Secundo, Elena, Martinaitis, \& Leitner, 2017; Bratianu, 2018a). Además, el capital estructural (CE) en la instancia de posesión de manuales de procedimientos por parte de la universidad indica la relevancia de la cultura organizacional para el adecuado cumplimiento de las funciones 
de la organización universitaria (Blankenburg, 2018, pp. 16; Cricelli, Greco, Grimaldi \& Llanes, 2018; Mercado, Gil y Cernas, 2016b).

De acuerdo con lo anterior, el conjunto de UCE en lo concerniente a la diseminación de la información, almacenamiento, apropiación y el compromiso con el conocimiento promueven en gran medida los procesos particulares de creación (CC), transferencia/almacenamiento (TA) y aplicación/uso (AU) del conocimiento de la universidad. Así, el proceso de GC que efectúan las UCE manifiesta una correlación con el resultado del proceso de gestión interno denominado $\mathrm{Cl}$, tanto en la experiencia del $\mathrm{CH}$ como en el CE y la infraestructura física (véase gráfica 6). Por lo tanto, se confirma que los procesos particulares de gestión del conocimiento de CC, TA, AU generan los elementos del capital intelectual en el conjunto de Universidades Cofinanciadas del Ecuador (UCE), como lo sugieren Govender, Perumal \& Perumal (2018).

\section{Conclusiones y recomendaciones}

El Modelo para la Evaluación de la Gestión del Conocimiento de la Universidad (MEGCU), propuesto en este documento, es operacionalizado por medio del Cuestionario de Evaluación de la Gestión del Conocimiento Universitario (CEGCU) y que se encuentra compuesto por 80 ítems (41 ítems del proceso de gestión del conocimiento y 39 ítems del capital intelectual) para la evaluación a la universidad (Cuadrado, 2017).

Al considerar a la gestión del conocimiento como un proceso continuo y dinámico que promueve el desarrollo de la universidad y al capital intelectual como el resultado de los procesos gestión internos realizada por la organización universitaria, la evaluación tanto de la GC, como del $\mathrm{Cl}$ de la universidad a partir de un modelo se convierte en una actividad de carácter prioritaria al encontrarse como base de la planificación, estructuración de planes, programas y actividades de la institución.

La evidencia empírica obtenida muestra que los procesos particulares de GC generan los elementos del $\mathrm{Cl}$ del conjunto de Universidades Cofinanciadas del Ecuador (UCE). De esta forma, la creación de conocimiento (CC) se encontró como elemento base para obtener los resultados de conocimiento $\mathrm{Cl}$. Como puede verse, la necesidad de la ejecución de procesos de gestión del conocimiento que manifiestan las UCE deja ver el reto del compromiso institucional para la actualización de procesos internos.

En concordancia con lo anterior, el compromiso con el conocimiento de los docentes, la transferencia y almacenamiento del conocimiento 
se encuentran como los principales factores condicionantes para los niveles de gestión alcanzados por las UCE. Además, se confirma que las universidades que efectúan los procesos de aplicación/uso de conocimiento $(\mathrm{AU})$ y transferencia/almacenamiento de conocimiento (TA) alcanzan resultados de conocimiento más altos.

De esta forma, tras la prueba del objeto de estudio complejo en el contexto Latinoamericano de siete Universidades cofinanciadas del Ecuador (UCE), se presenta al Modelo para la Evaluación de la Gestión del Conocimiento de la Universidad (MEGCU) como un aporte a la Teoría de Recursos y Capacidades de la Administración (TRC), a la Disciplina emergente de Gestión del Conocimiento (GC) y a la Teoría de Creación del Conocimiento Organizacional (TCO).

Camino, sobre tu piel morena y siento tu latido y miro todo lo bueno que los dos hemos vivido; te digo: sólo hay razones para estar agradecidos, eso que somos y lo que siempre hemos sido.

Amigos, porque ganamos cuando mucho hemos perdido somos lo mismo, porque peleamos contra el mismo enemigo, yo sigo hasta el final y tú siempre estás conmigo. hemos caído, pero no nos han vencido! Yo soy ecuatoriano, sí señor, y tú eres mi Ecuador, Ecuador ecuatoriano, con un solo corazón. eres mi alma ecuatoriana, soy tu sangre y tu color somos dos enamorados, tú mi tierra, yo tu flor.

Hoy te canto ecuatoriano, porque soy del Ecuador, Ecuador ecuatoriano, tú mi vida, yo tu voz, Ecuador ecuatoriano, piel de luna, piel de sol. Ansío seguir forjando a tu lado mi destino tú conmigo, empujando nuestros sueños y delirios país querido, siempre juntos siempre alegres, siempre unidos, no hay espacio para el miedo ni el olvido.

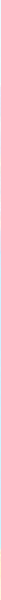


Gráfica 6. La GC y el Cl en el conjunto de universidades cofinanciadas del Ecuador

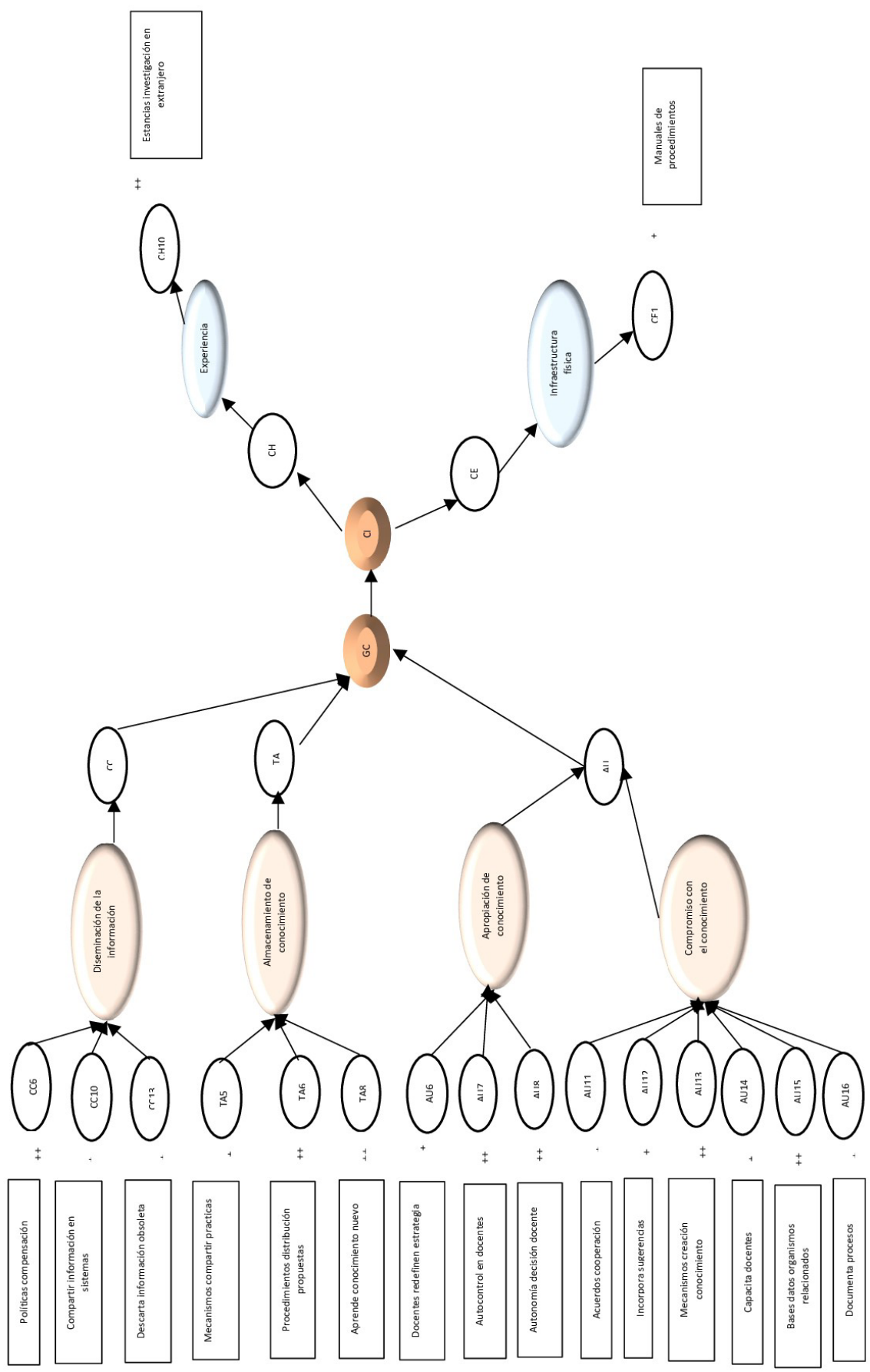

Fuente: Elaboración propia 


\section{Referencias bibliográficas}

1. Abbas, A., Avdic, A., Xiaobao, P., Hasan, M., \& Ming, W. (2019). Universitygovernment collaboration for the generation and commercialization of new knowledge for use in industry. Journal of Innovation \& Knowledge, 4 (1), 23-31. https://doi.org/10.1016/j.jik.2018.03.002

2. Askary, S.; Qayyum, N. \& Van Sant, R. (2015). Culture, communication skills and intellectual capital: a theoretical framework. International Journal of Higher Education and Sustainability, 1 (1), 88-101.

3. Alimohammadlou, M., \& Eslamloo, F. (2016). Relationship between Total Quality Management, Knowledge Transfer and Knowledge Diffusion in the Academic Settings. Procedia - Social and Behavioral Sciences, 230, 104-111. https://doi. org/10.1016/j.sbspro.2016.09.013

4. Azagra-Caro, J., Barberá-Tomás, D., Edwards-Schachter, M., \& Tur, E. (2017). Dynamic interactions between university-industry knowledge transfer channels: A case study of the most highly cited academic patent. Research Policy, 46 (2), 463-474. https://doi. org/10.1016/j.respol.2016.11.011

5. Baglieri, D., Baldi, F., \& Tucci, C. (2018). University technology transfer office business models: One size does not fit all. Technovation, 76-77, 51-63. https://doi.org/10.1016/j. technovation.2018.05.003

6. Barley, W., Treem, J., \& Kuhn, T. (2018). Valuing Multiple Trajectories of Knowledge: A Critical Review and Agenda for Knowledge Management Research. Academy of Management Annals, 12 (1), 278-317. https://doi.org/10.5465/annals.2016.0041

7. Bejinaru, R. (2017a). Dynamic Capabilities of Universities in the Knowledge Economy. Management Dynamics in the Knowledge Economy, 5 (4), 577-595. https://doi. org/10.25019/MDKE/5.4.07

8. Bejinaru, R. (2017b). Knowledge strategies aiming to improve the intellectual capital of universities. Management \& Marketing, 12 (3), 500-523. https://doi.org/10.1515/ mmcks-2017-0030

9. Bejinaru, R. (2018). The key processes of knowledge dynamics and intellectual capital in organizations. In C. Brătianu, A. Zbuchea, \& A. Vițelar (Eds.), Strategica 2018. Challenging the Status Quo in Management and Economics (pp. 599-609). Bucharest: Faculty of Management (SNSPA).

10. Blind, Knut, Pohlisch, J., \& Zi, A. (2018). Publishing, patenting, and standardization: Motives and barriers of scientists. Research Policy, 47 (7), 1185-1197. https://doi. org/10.1016/j.respol.2018.03.011

11. Blankenburg, K. (2018). Intellectual Capital in German Non-profit Organisations (First). In Contributions to Management Science (First). https://doi.org/10.1007/978-3-319-62655-0

12. Bratianu, C. (2018a). A holistic view of the organizational knowledge dynamics. HOLISTICA - Journal of Business and Public Administration, 9 (2), 7-22. https://doi. org/10.2478/hjbpa-2018-0009

13. Bratianu, C. (2018b). Universities as learning organizations: challenges and strategies. In C. Brătianu, A. Zbuchea, \& A. Vițelar (Eds.), Strategica 2018. Challenging the Status Quo in Management and Economics (pp. 545-554). Bucharest: Faculty of Management (SNSPA). 
14. Cordero, D. (2016). Modelo para Gobierno de Tecnologías de la Información (GTI): caso de las Universidades Cofinanciadas de la Zona 6 de la República del Ecuador. Tesis doctoral, Universidad Nacional Autónoma de México, pp. 315.

15. Creswell, J. (2014). Research Design. Qualitative, Quantitative, and Mixed Methods Approaches. California: Sage Publications.

16. Crescenzi, R., Nathan, M., \& Rodríguez-Pose, A. (2016). Do inventors talk to strangers? On proximity and collaborative knowledge creation. Research Policy, 45 (1), 177-194. https://doi.org/10.1016/j.respol.2015.07.003

17. Cricelli, L., Greco, M., Grimaldi, M., \& Llanes Dueñas, L. (2018). Intellectual capital and university performance in emerging countries. Journal of Intellectual Capital, 19 (1), 71-95. https://doi.org/10.1108/JIC-02-2017-0037

18. Cuadrado, G. y Carrillo, P. (2016). Los procesos de gestión del conocimiento. El caso de las universidades del Ecuador. En: Universidad Autónoma de Yucatán (Ed.), XX Congreso internacional de investigación en ciencias administrativas (ACACIA) (p. 30). Merida, Yucatán: Universidad Autónoma de Yucatán.

19. Cuadrado, G. (2017). Modelo para la evaluación de la gestión del conocimiento de la universidad: el caso de la universidad cofinanciada del Ecuador. Universidad Nacional Autónoma de México (UNAM).

20. Demuner, M.; Nava, R. \& Ibarra, M. (2016). Dimensiones e indicadores de capital estructural para la universidad pública. In M. Nava, Rosa; Mercado, Patricia; Demuner (Ed.), El capital intelectual en la universidad pública (1st ed., pp. 61-80). Ciudad de Méjico: Universidad Autónoma del Estado de México.

21. De Silva, M., \& Rossi, F. (2018). The effect of firms' relational capabilities on knowledge acquisition and co-creation with universities. Technological Forecasting and Social Change, 133, 72-84. https://doi.org/10.1016/j.techfore.2018.03.004

22. Ecuador. Presidencia de la República. Ley Orgánica de Educación Superior (LOES) (2018) Quito.

23. Elezi, E., \& Bamber, C. (2018). Knowledge management in the uk higher education institutions: what type of outcomes do higher education partnerships attain? In C. Brătianu, A. Zbuchea, \& A. Vițelar (Eds.), Strategica 2018. Challenging the Status Quo in Management and Economics (pp. 573-586). Bucharest: Faculty of Management (SNSPA).

24. Etzkowitz, H. (2017). Innovation Lodestar: The entrepreneurial university in a stellar knowledge firmament. Technological Forecasting and Social Change, 123, 122-129. https://doi.org/10.1016/j.techfore.2016.04.026

25. Ferreira, J., Mueller, J., \& Papa, A. (2018). Strategic knowledge management: theory, practice and future challenges. Journal of Knowledge Management, JKM-07-20180461. https://doi.org/10.1108/JKM-07-2018-0461

26. Frondizi, R., Fantauzzi, C., Colasanti, N., \& Fiorani, G. (2019). The Evaluation of Universities' Third Mission and Intellectual Capital: Theoretical Analysis and Application to Italy. Sustainability, 11 (12), 3455. https://doi.org/10.3390/su11123455

27. Galego-Álvarez, I., Formigoni, H., y Antunes, M. (2014). Corporate Social Responsibility Practices at Brazilian Firms. Revista de Administração de Empresas, 54 (1), 12-27.

28. García-Berro, E., Roca, S., Navallas, F., Soriano, M., y Ras, A. (2016). El impacto de las políticas de evaluación del profesorado en la posición en los ránquines universitarios: el caso de la Universidad Politécnica de Cataluña. Aula Abierta, 44 (1), 23-30. 
29. Govender, L., Perumal, R, \& Perumal, S. (2018). Knowledge management as a strategic tool for human resource management at higher education institutions. SA Journal of Information Management, 20 (1). https://doi.org/10.4102/sajim.v20i1.966

30. Gupta, P., Mehrotra, D., y Sharma, T. (2015). Identifying Knowledge Indicators in Higher Education Organization. Procedia Computer Science, 46, 449-456. https://doi. org/10.1016/j.procs.2015.02.043

31. Henríquez, P. (2018). El papel estratégico de la educación superior en el desarrollo sostenible de América Latina y el Caribe. In H. Henríquez, Pedro; Juri (Ed.), Tendencias de la educación superior en América Latina y el Caribe 2018 (Primera, pp. 275-308). Córdoba, Argentina: UNESCO-IESALC.

32. Lara, F. (2017). Las Ciencias de la Complejidad en la Solución de Nuestros Problemas Sociales. In Aplicaciones de las ciencias de la complejidad al diagnóstico e intervención en problemas sociales (pp. 1-41). Ciudad de Méjico: Ediciones Académicas de Pedagogía Colofon.

33. Leih, S., \& Teece, D. (2016). Campus Leadership and the Entrepreneurial University: A Dynamic Capabilities Perspective. Academy of Management Perspectives, 30 (2), 182-210. https://doi.org/10.5465/amp.2015.0022

34. Mahdi, O., Nassar, I., \& Almsafir, M. (2019). Knowledge management processes and sustainable competitive advantage: An empirical examination in private universities. Journal of Business Research, 94, 320-334. https://doi.org/10.1016/j. jbusres.2018.02.013

35. Mansor, Z., Mustaffa, M. \& Salleh, L. (2015). Motivation and Willingness to Participate in Knowledge Sharing Activities Among Academics in a Public University. Procedia Economics and Finance, 31, 286-293. https://doi.org/10.1016/S2212-5671(15)01188-0

36. Marchiori, D., \& Franco, M. (2019). Knowledge transfer in the context of interorganizational networks: Foundations and intellectual structures. Journal of Innovation \& Knowledge. https://doi.org/10.1016/j.jik.2019.02.001

37. Medland, E. (2016). Assessment in higher education: drivers, barriers and directions for change in the UK. Assessment \& Evaluation in Higher Education, 41 (1), 81-96. https:// doi.org/10.1080/02602938.2014.982072

38. Mercado, P.; García, P. \& Cernas, D. (2016a). El capital intelectual en la gestión de la universidad pública: un medio para sus fines. In M. Nava, Rosa; Mercado, Patricia; Demuner (Ed.), El capital intelectual en la universidad pública (1st ed., pp. 19-40). Ciudad de Méjico: Universidad Autónoma del Estado de México.

39. Mercado, P., Gil-Monte, P., \& Cernas, D. (2016b). Validez Inicial de una Escala de Medición del Capital Intelectual en Universidades. Universitas Psychologica, 15 (2), 109. https://doi.org/10.11144/Javeriana.upsy15-2.viem

40. Miake, A., Carvalho, R., Pinto, M., \& Graeml, A. (2018). Customer Knowledge Management (CKM): Model Proposal and Evaluation in a Large Brazilian Higher Education Private Group. Brazilian Business Review, 15 (2), 135-151. https://doi. org/10.15728/bbr.2018.15.2.3

41. Mochales, G. (2014). Modelo explicativo de la responsabilidad social corporativa estratégica. Tesis Doctoral, Universidad Complutense de Madrid, pp. 335.

42. Muscio, A., \& Ramaciotti, L. (2019). How does academia influence Ph.D. entrepreneurship? New insights on the entrepreneurial university. Technovation, 8283, 16-24. https://doi.org/10.1016/j.technovation.2019.02.003 
43. Nava, R.; Cernas, D.; Jiménez, P. (2016). Modelo de medición de capital relacional para la universidad pública. In M. Nava, Rosa; Mercado, Patricia; Demuner (Ed.), El capital intelectual en la universidad pública (1 ${ }^{\text {a }}$ ed., pp. 81-100). Ciudad de Méjico: Universidad Autónoma del Estado de México.

44. Nonaka, I. \& Takeuchi, H. (1995). The Knowledge Creating Company. New York: Oxford University Press.

45. Olcay, G., \& Bulu, M. (2017). Is measuring the knowledge creation of universities possible?: A review of university rankings. Technological Forecasting and Social Change, 123, 153-160. https://doi.org/10.1016/j.techfore.2016.03.029

46. Ostrom, E. (2015). Comprender la diversidad institucional. Méjico, D. F.: Fondo de Cultura Económica, Universidad Autónoma Metropolitana.

47. Petrova, G., Smokotin, V., Kornienko, A., Ershova, I. A., \& Kachalov, N. A. (2015). Knowledge Management as a Strategy for the Administration of Education in the Research University. Procedia - Social and Behavioral Sciences, 166, 451-455. https:// doi.org/10.1016/j.sbspro.2014.12.552

48. Ramírez, Luis (2016). La evaluación de la educación superior: Un campo de tensiones. Fides Et Ratio, 11 (11), 125-138.

49. Ramírez-Córcoles, Y., \& Manzaneque-Lizano, M. (2015). The relevance of intellectual capital disclosure: empirical evidence from Spanish universities. Knowledge Management Research \& Practice, 13 (1), 31-44. https://doi.org/10.1057/kmrp.2013.27

50. Ramírez-Córcoles, Y.; Manzaneque-Lizano, M., \& Priego, A. (2017). Formulating and elaborating a model for the measurement of intellectual capital in Spanish public universities. International Review of Administrative Sciences, 83 (1), 149-176. https:// doi.org/10.1177/0020852315575168

51. Rinesi, E. (2018). Universidad y desarrollo (Notas sobre la cuestión del "derecho a la Universidad"). In L. Petersen (Ed.), Balances y desafíos hacia la CRES 2018. Cuaderno 1. Aportes para pensar la Universidad Latinoamericana (1a. ed., pp. 61-72). Buenos Aires: Consejo Latinoamericano de Ciencias Sociales (CLACSO).

52. Sadeghi B., S., Hasani, K., \& Delshab, V. (2019). Investigating the influence of knowledge management on organizational innovation in higher educational institutions. Kybernetes, K-09-2018-0492. https://doi.org/10.1108/K-09-2018-0492

53. Secundo, Giustina, Elena-Pérez, S., Martinaitis, Ž., \& Leitner, K. (2017). An Intellectual Capital framework to measure universities' third mission activities. Technological Forecasting and Social Change, 123, 229-239. https://doi.org/10.1016/j. techfore.2016.12.013

54. Santos De Souza, B. (2015). La universidad en el Siglo XXI (1 a ed.). Méjico, D. F.: Siglo XXI Editores.

55. Serenko, A., \& Bontis, N. (2016). Negotiate, reciprocate, or cooperate? The impact of exchange modes on inter-employee knowledge sharing. Journal of Knowledge Management, 20 (4), 687-712. https://doi.org/10.1108/JKM-10-2015-0394

56. Serenko, A. (2019). Looking Beyond the Pointing Finger: Ensuring the Success of the Scholarly Capital Model in the Contemporary Academic Environment. Communications of the Association for Information Systems, 217-226. https://doi. org/10.17705/1CAIS.04413 
57. Sengupta, A., \& Ray, A. (2017). University research and knowledge transfer: A dynamic view of ambidexterity in british universities. Research Policy, 46 (5), 881-897. https:// doi.org/10.1016/j.respol.2017.03.008

58. Streck, D. (2018). La universidad como lugar de posibilidades. In L. K. Suasnábar, C.; Del Valle, D. \& Didriksson, A. (Ed.), Balances y desafíos hacia la CRES 2018 (1ª. ed., pp. 131 - 137). Buenos Aires: Instituto de Estudios y Capacitación de la Federación Nacional de Docentes Universitarios (IEC-CONADU), Consejo Latinoamericano de Ciencias Sociales (CLACSO), Universidad Nacional de las Artes (UNA).

59. Schaik, P. van, Volman, M., Admiraal, W. \& Schenke, W. (2018). Barriers and conditions for teachers' utilisation of academic knowledge. International Journal of Educational Research, 90, 50-63. https://doi.org/10.1016/j.ijer.2018.05.003

60. Zharova, A. (2018). Measures of University Research Output. Humboldt-Universität zu Berlin.

Para citar este artículo:
Cuadrado-Barreto, G. (2020). La gestión del conocimiento de la universidad cofinanciada del Ecuador (UCE). Teuken Bidikay 11 (17), 109130. doi: 10.33571/teuken.v11n17a6

Ge: HJS.

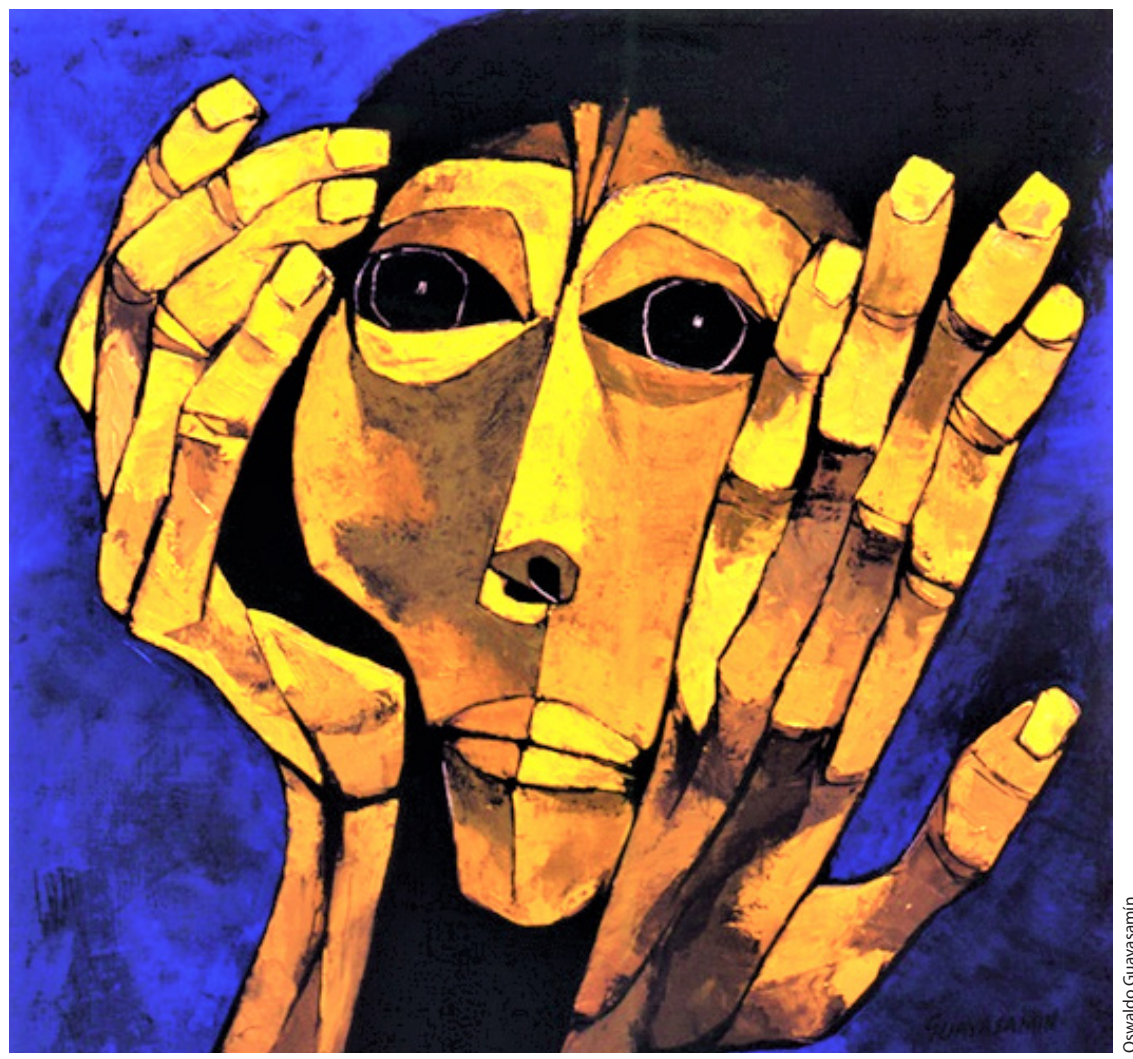

Teuken Bidikay Vol. 11 № 17 (Medellín, Colombia) * Jul-Dic 2020. ISSN: 2215-8405 - e-ISSN 2619-1822. Pp. 109-130 